\title{
Cerebrospinal Fluid Leakage after Surgeries on the Thoracic Spine: A Review of 362 Cases
}

\author{
Panpan $\mathrm{Hu}^{*}$, Miao Yu*, Xiaoguang Liu, Zhongjun Liu, Liang Jiang, Feng Wei, Zhongqiang Chen \\ Department of Orthopedics, Peking University Third Hospital, Beijing, China
}

\section{Study Design: A retrospective clinical review.}

Purpose: To describe the incidence of cerebrospinal fluid leakage (CSFL) after thoracic decompression and examine the CSFL predisposing clinical factors.

Overview of Literature: CSFL is a common complication following thoracic decompression but has not been sufficiently addressed in former studies.

Methods: A cohort of 362 cases of thoracic decompression from February of 2005 to June of 2013 was examined. The case medical records were reviewed and the occurrence of CSFL and the related clinical parameters were noted. The incidence of CSFL for the entire cohort and each surgical approach were described. Besides, the relationship between CSFL and other clinical parameters were assessed, of which odds ratio values of all CSFL-associated parameters were calculated using multivariate logistic regression analysis. Results: The incidence of CSFL for the entire cohort was 32.3\%. Different surgical approaches had different incidences of CSFL, and circumferential decompression had the highest incidence. Though many different clinical parameters were related to the occurrences of CSFL, being older than 52 years, having ossification of the posterior longitudinal ligament or having longer operative segments than 3 vertebrae were significant risk factors for CSFL $(p<0.05)$. Besides, surgeries on the mid-thoracic spine had an increased risk of CSFL $(p<0.05)$.

Conclusions: From our analysis, CSFL was a common complication after thoracic decompression with the incidence of up to $32.3 \%$. This study identified the predisposing clinical factors, and spinal surgeons should be aware of these risk factors to reduce its incidence.

Keywords: Cerebrospinal fluid leakage; Thoracic decompression; Incidence; Risk factors

\section{Introduction}

Surgery on the thoracic spine is a great challenge for spinal surgeons, and frequently complicated by undesirable events, such as cerebrospinal fluid leakage (CSFL). The CSFL incidences for cervical and lumbar spine had been characterized [1-4], but those for thoracic spine still remain to be clearly described. Though there were many publications describing CSFL after surgeries on the whole spine [5-8], the reports either had a small sample for thoracic decompression or did not list the incidence for each spinal segment. Also reports focusing on thoracic decompression only included relatively small sample sizes, which led to apparent inconsistencies for the CSFL incidences among the reports. For example, Yamazaki et al. [9] reported that 1 out of 24 patients with thoracic myelopathy

\footnotetext{
Received Sep 12, 2015; Revised Oct 12, 2015; Accepted Oct 12, 2015

Corresponding author: Xiaoguang Liu

Department of Orthopedics, Peking University Third Hospital. No. 49 Huayuanbei Rd, Haidian District, Beijing 100191, China

Tel: +86-010-82267368, Fax: +86-010-82267368, E-mail: puth_lxg@163.com

*P. Hu and M. Yu are equal contributors and share the first authorship.
} 
Table 1. Surgical approaches for different clinical scenarios ${ }^{\text {a) }}$

\begin{tabular}{ll}
$\begin{array}{l}\text { Surgical approaches } \\
\text { Circumferential decompression }\end{array}$ & \multicolumn{1}{c}{ Recommended clinical scenarios } \\
Posterior decompression & Posterior compression with or without anterior flat-type compression \\
\hline Anterior decompression & $\begin{array}{l}\text { Anterior regional beak-type compression at mid- and lower thoracic spine without posterior } \\
\text { compression }\end{array}$ \\
\hline
\end{tabular}

${ }^{\text {a) }}$ Suitable for the majority of the cohort but allowing for exceptions for whom another surgical approach was finally performed.

caused by ossification of the posterior longitudinal ligament (OPLL) developed CSFL, while Min et al. [10] noted 10 out of 20 patients with thoracic OPLL had this complication. There have also been a few studies having larger sample sizes, but these merely recruited patients with a certain pathology, such as OPLL, or undergoing a single surgical approach. For example, Matsumoto et al. [11] undertook a multi-institutional study involving 154 patients but only recruited those with thoracic OPLL, and Sun et al. [12] reviewed 266 patients with thoracic ossification of ligamentum flavum (OLF) for whom en bloc laminectomy was the exclusive surgical approach. Therefore, there has been a need for a comprehensive investigation of CSFL following surgeries on the thoracic spine for a relatively large series with different surgical approaches. This study was performed to meet the above requirements by reviewing 362 cases of thoracic decompression, and it aimed to review the incidence of CSFL after thoracic surgeries and its predisposing clinical parameters.

\section{Materials and Methods}

\section{Patient population}

The patients included in the study were diagnosed with a degenerative thoracic spinal disease and underwent decompression by experienced surgeons in our hospital from February 2005 to June 2013. Patients were excluded if they: (1) did not have complete medical records including those with previous spinal surgeries with inaccessible data from other hospitals; (2) had a history of trauma or non-surgical invasive manipulations on the thoracic spine, such as percutaneous vertebral biopsy; (3) had a history of other diseases which compromised the dural sac, such as spinal tumors; and (4) had received a combination of cervical or lumbar decompression at the same time. This study was approved by our Institutional Review Board (IRB) and was supported by two governmental grants (see the "Acknowledgments").

After the exclusions, a total of 362 consecutive cases of thoracic decompression in 345 patients were included in the study. There were 17 patients who underwent two thoracic decompressions and included 5 patients whose second decompression involved formerly operated segments.

\section{Surgical procedures}

In general, all the recruited operations could be categorized into three surgical approaches: posterior decompression (PD) via en bloc laminectomy with or without fusion, circumferential decompression $(\mathrm{CD})$ via a transpedicular approach [13] and anterior decompression (AD) via an extrapleural (extrapleuroperitoneal) or transthoracic approach. Imaging was the main factor used in selection of a suitable surgical approach for each patient (Table 1). Particularly, PD was the initial surgical approach for all 5 cases of revision surgeries. After an average interval of 55.6 months (ranging from 3 months to 11 years), the revision surgeries were undertaken with transpedicular $\mathrm{CD}$ manipulations in 4 cases, with or without the extension of decompression segments, and extrapleuroperitoneal $\mathrm{AD}$ manipulation in 1 case on the previously operated spinal segments. In total, there were 244 cases for PD, 79 for CD and 39 for $\mathrm{AD}$ procedures.

\section{Definition of CSFL}

The diagnosis of CSFL was made according to the following three criteria: (1) dural tears or appearance of clear fluid during the operation; (2) persistent drain of clear fluid from subfascial tubes; and (3) subcutaneous collection of clear fluid confirmed by puncture or aspiration.

\section{Information recorded}

The information collected included patient demographic 
Table 2. Incidences of cerebrospinal fluid leakage for the entire cohort and each surgical approach

\begin{tabular}{lccc} 
Groups & Total cases & Leakage cases & Incidence (\%) \\
\hline All & 362 & 117 & 32.3 \\
Circumferential decompression & 79 & 33 & 41.8 \\
\hline Posterior decompression & 244 & 76 & 31.1 \\
Anterior decompression & 39 & 8 & 20.5 \\
\hline
\end{tabular}

data, coexisting diseases, preoperative duration of neurological symptoms and preoperative diagnoses. Imaging work-ups were reviewed and the sources of compression were recorded. Operation notes and anesthetic charts were retrieved and scrutinized to note the surgical approaches, operative duration, blood loss and operative segments. Drainage fluid from the subfascial tubes and emergence of subcutaneous fluid collection during postoperative hospital stay were also examined to determine whether CSFL occurred.

\section{Data processing and statistical analysis strategies}

All statistical analyses in this study were conducted in terms of operations rather than patients. IBM statistics SPSS ver. 20 (IBM Corp., Armonk, NY, USA) was used for statistical analysis. Descriptive statistics were presented in form of mean \pm standard deviation and the confidence interval was set at 95\% (95\% CI). The incidences of CSFL were separately decided for the entire cohort and each surgical approach. Relationships between different clinical parameters were decided by different methods, including unpaired student $t$ test (two-tailed), Pearson's $\chi^{2}$ test (twotailed) and Spearman's correlation test (two-tailed). For CSFL-associated clinical parameters, if they were recorded with continuous values, the receiver operating characteristic (ROC) curve was drawn for each parameter and the optimal cut-off value was decided. Subsequently, the odds ratio (OR) values for all CSFL-associated parameters were calculated using multivariate logistic regression analysis. Besides, OR value of each vertebra for CSFL was also calculated. Statistical significance was set at 0.05 .

\section{Results}

\section{Descriptive results for the entire cohort}

The cohort included 208 males and 154 females, with

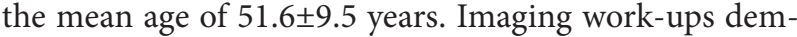
onstrated OPLL in 120 cases and other pathologies in 142 cases, such as OLF and herniated discs. There were 96 cases having a comorbidity of hypertension and 47 cases having diabetes mellitus. Mean values for preoperative symptom duration were $27.0 \pm 39.6$ months, the Japanese Orthopaedic Association (JOA) scores 5.4 \pm 2.5 , lengths of operative segments $3.8 \pm 1.8$, operative duration $191.5 \pm 72.9$ minutes and blood loss $1138.0 \pm 922.3 \mathrm{~mL}$.

\section{Incidence of CSFL for the entire cohort}

One hundred seventeen out of 362 recruited thoracic decompressions were complicated with CSFL, yielding an incidence of $32.3 \%$ for the entire cohort (Table 2 ). In particular, the diagnosis of CSFL was made during postoperative hospitalization in 13 cases, for which no dural tears were intraoperatively recognized. Also, all 5 cases of patients with previously operated segments, who received an extra decompression, developed CSFL.

\section{Incidences of CSFL for different surgical approaches}

There were 33 cases developing CSFL in the CD group, yielding an incidence of $41.8 \%$ for this group, which was higher than $31.1 \%$ for the PD group and $20.5 \%$ for the AD group (Table 3). Besides, the cases of missed diagnosis during the operation in three groups were 6,7 , and 0 , respectively, yielding a rate of $18.2 \%, 9.2 \%$ and 0 for each group.

\section{Predisposing clinical factors for CSFL}

Comparison analysis between the CSFL and non-CSFL subgroups demonstrated significant differences in age, preoperative JOA score, operative duration, blood loss and lengths of operative segments $(p<0.05)$ (Table 3$)$. There was, however, no difference in the duration of 
Table 3. Comparison between the groups with and without cerebrospinal fluid leakage

\begin{tabular}{lccc} 
Items & Leakage $(\mathrm{n}=117)$ & Non-leakage $(\mathrm{n}=245)$ & $p$-value \\
\hline Age $(\mathrm{yr})$ & $53.9 \pm 9.7$ & $50.5 \pm 9.3$ & $0.001^{\mathrm{a})}$ \\
Symptom duration $(\mathrm{mo})$ & $23.4 \pm 34.8$ & $28.7 \pm 41.7$ & 0.230 \\
\hline Preoperative JOA score & $4.9 \pm 2.3$ & $5.7 \pm 2.5$ & $0.005^{\mathrm{a})}$ \\
Operative segments & $4.4 \pm 1.8$ & $3.5 \pm 1.7$ & $0.000^{\mathrm{a}}$ \\
\hline Operative duration (min) & $208.6 \pm 68.5$ & $183.3 \pm 73.6$ & $0.002^{\mathrm{a})}$ \\
\hline Blood loss (mL) & $1308.7 \pm 1104.4$ & $1056.5 \pm 865.8$ & $0.019^{\text {a) }}$ \\
\hline
\end{tabular}

Values are presented as mean \pm standard deviation.

JOA, Japanese Orthopaedic Association.

${ }^{\text {a) }}$ Statistically significant at $p<0.05$, unpaired student $t$ test (two-tailed).

preoperative symptoms $(p=0.230$ ) (Table 3 ) for the two groups. According to the ROC curve for each parameter and CSFL, the optimal cut-off values of these parameters were determined (Table 4).

This analysis found that for patients older than $52(\mathrm{OR}$, 1.688; 95\% CI, 1.029-2.770; $p=0.038$ ), having OPLL (OR, 2.496; 95\% CI, 1.368-4.553; $p=0.003$ ) or having operative segments longer than 3 vertebrae (OR, 1.725; 95\% CI, 1.008-2.952; $p=0.047$ ), there were a significant risk of CSFL (Table 4). Besides, vertebrae T5 to T7 had a higher OR than other vertebrae (Fig. 1).

\section{Discussion}

This study found that the incidence of CSFL after thoracic decompression was $32.3 \%$, but varied significantly across different surgical approaches (Table 2). This surgery

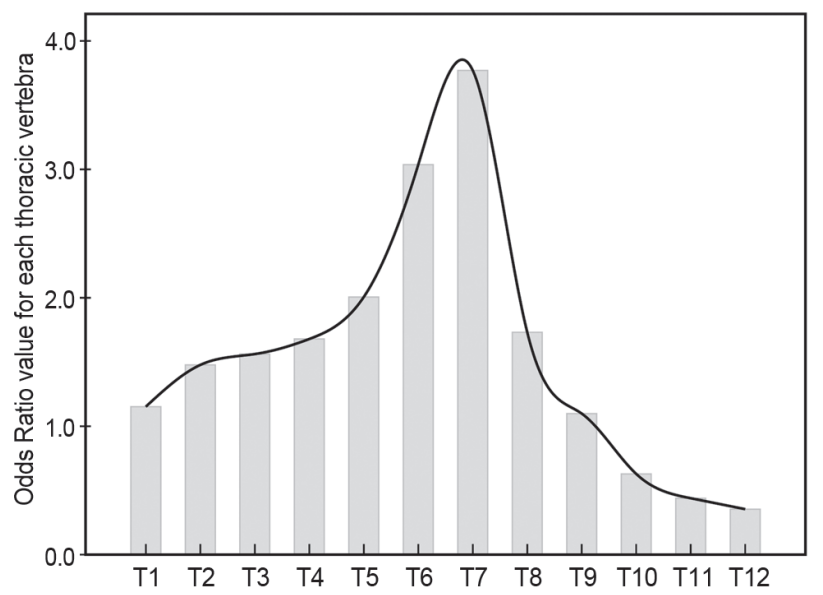

Fig. 1. Diagram displaying odds ratio value of each thoracic vertebra for cerebrospinal fluid leakage. It showed that $\mathrm{T5}$ (odds ratio [OR], 2.01), T6 (OR 3.01) and T7 (OR 3.77) had higher OR values than other thoracic vertebrae. approach-associated study of the incidence of CSFL had not been clearly reported in former publications $[11,14$ 17]. Yamazaki et al. [17] reported that the respective CSFL incidences for $\mathrm{AD}, \mathrm{CD}$ and $\mathrm{PD}$ in a study of 51 patients with thoracic OPLL and reminded that operations including anterior manipulations had a higher risk for CSFL. Their study, however, only involved a limited sample size and did not provide detailed operative data. This present study found that $\mathrm{CD}$ had a significantly higher likelihood of CSFL, accounting for $41.8 \%$ of the CD subgroup (Table 2). This proportion was quite high, compared with previous publications on the same procedure [17-19]. This may be due to both pathological and technical reasons. Pathologically, nearly all of CD procedures in this study were performed in patients with thoracic OPLL, which was identified as an essential risk factor for CSFL (Table 4) as there is a higher occurrence of dural adhesion in patients with OPLL than other pathologies $[10,16,20]$. Technically, there is a partial or entire loss of visual supervision during anterior manipulations due to the blockage of the spinal cord, which may increase the risk of dural tears during the procedure. This shortcoming also contributed to the higher proportion of CSFL cases being intraoperatively unrecognized in $\mathrm{CD}$ subgroup. Generally, the emergence of clear fluid in the operative field was remarkable in alerting to dural tears in cases of lost visual supervision, but not for tiny tears, through which the egress of cerebrospinal fluid was slow and minimal. As the procedure is also accompanied by constant and plentiful bleeding, the signs of CSFL may go unnoticed during surgery. Floating and extirpation of OPLL are both accepted during CD procedure and the latter has been much more common in our hospital, aiming to avoid the potential compromise from residual ossification on the dural sac, which was 
Table 4. Analysis of clinical predisposing factors for CSF leakage

\begin{tabular}{|c|c|c|c|c|c|c|}
\hline \multirow{3}{*}{ ltems } & \multicolumn{3}{|c|}{ Analysis for individual clinical factors ${ }^{\mathrm{a})}$} & \multicolumn{3}{|c|}{ Analysis for all the associated clinical factors ${ }^{\mathrm{b})}$} \\
\hline & \multicolumn{2}{|c|}{ CSF leakage } & \multirow[t]{2}{*}{$p$-value } & \multicolumn{2}{|r|}{ Odds ratio } & \multirow[t]{2}{*}{$p$-value } \\
\hline & Cases & Incidence $(\%)$ & & Value & 95\% Confidence interval & \\
\hline Age (yr) & & & $0.010^{\mathrm{al}}$ & 1.688 & $(1.029,2.770)$ & $0.038^{b)}$ \\
\hline$\leq 52(n=182)$ & 47 & 25.8 & & & & \\
\hline$>52(n=180)$ & 70 & 38.9 & & & & \\
\hline Sex & & & $0.023^{\mathrm{al}}$ & 1.267 & $(0.773,2.076)$ & 0.348 \\
\hline Males (n=208) & 57 & 27.4 & & & & \\
\hline Females ( $n=154)$ & 60 & 39.0 & & & & \\
\hline Hypertension & & & $0.030^{\mathrm{al}}$ & 1.210 & $(0.690,2.122)$ & 0.506 \\
\hline No $(n=266)$ & 77 & 28.9 & & & & \\
\hline Yes (n=96) & 40 & 41.7 & & & & \\
\hline Diabetes mellitus & & & $0.029^{a l}$ & 1.410 & $(0.704,2.824)$ & 0.333 \\
\hline No $(n=315)$ & 95 & 30.2 & & & & \\
\hline Yes (n=47) & 22 & 46.8 & & & & \\
\hline Symptom duration (mo) & & & 0.911 & & Excluded & \\
\hline$\leq 10(n=175)$ & 56 & 32.0 & & & & \\
\hline$>10(n=187)$ & 61 & 32.6 & & & & \\
\hline Pathology & & & $0.000^{\mathrm{a})}$ & 2.496 & $(1.368,4.553)$ & $0.003^{\mathrm{bl}}$ \\
\hline Non-OPLL (n=242) & 60 & 24.8 & & & & \\
\hline OPLL (n=120) & 57 & 47.5 & & & & \\
\hline Preoperative JOA score & & & $0.037^{\mathrm{a})}$ & 1.220 & $(0.719,2.070)$ & 0.461 \\
\hline$\geq 7(n=133)$ & 34 & 25.6 & & & & \\
\hline$<7(n=229)$ & 83 & 36.2 & & & & \\
\hline Surgical approaches & & & $0.042^{\mathrm{al}}$ & 0.591 & $(0.294,1.189)$ & 0.140 \\
\hline Non-CD (n=283) & 84 & 29.7 & & & & \\
\hline $\operatorname{CD}(n=79)$ & 33 & 41.8 & & & & \\
\hline Operative segments & & & $0.000^{\mathrm{al}}$ & 1.725 & $(1.008,2.952)$ & $0.047^{b)}$ \\
\hline$\leq 3(n=193)$ & 44 & 22.8 & & & & \\
\hline$>3(n=169)$ & 73 & 43.2 & & & & \\
\hline Operative duration (min) & & & $0.001^{\mathrm{a})}$ & 1.298 & $(0.708,2.380)$ & 0.399 \\
\hline$\leq 200(n=215)$ & 55 & 25.6 & & & & \\
\hline$>200(n=147)$ & 62 & 42.2 & & & & \\
\hline Blood loss (mL) & & & $0.004^{\mathrm{al}}$ & 1.095 & $(0.590,2.033)$ & 0.773 \\
\hline$\leq 650(n=147)$ & 35 & 23.8 & & & & \\
\hline$>650(n=215)$ & 82 & 38.1 & & & & \\
\hline
\end{tabular}

CSF, stands for cerebrospinal fluid; OPLL, ossification of posterior longitudinal ligament; JOA, Japanese Orthopaedic Association; CD, circumferential decompression.

${ }^{a)}$ Accomplished via Pearson's $\chi^{2}$ test (two-tailed), and $p<0.05$ indicates clinical factors associated with CSF leakage; ${ }^{\text {b) }}$ Accomplished via multiple logistic regression analysis, and $p<0.05$ indicates significant clinical predictors for CSF leakage. 
demonstrated in a multi-institutional research by Matsumoto et al. [11]. However, the dissection of OPLL from the adhesive dural sac also increases the risk of CSFL. Correspondingly, in this present study 66 of $79 \mathrm{CD}$ procedures were accomplished with the extirpation of OPLL, of which CSFL occurred in 35 cases, accounting for $53.0 \%$, which was higher than $30.8 \%$ in the floating subgroup.

Previous studies, though most of which were not conducted in patients with thoracic decompression, proved that older age, massive blood loss and longer operative segments were associated with a higher risk of CSFL [1,4$6,11,12]$. These associations were also confirmed in the present study, as well as in females, patients with lower preoperative JOA scores, having hypertension and diabetes mellitus (Table 4). This study also identified the optimal cut-off values for these clinical factors by employing ROC curves. The multivariate logistic regression analysis of all the CSFL-associated parameters, cases with age older than 52 years, having OPLL or with operative segments longer than 3 vertebrae had a significantly higher risk of CSFL (Table 4). As such, these three parameters could be recognized as the essential risk factors for CSFL. We also found that the operations involving mid-thoracic spine were more likely to develop CSFL (Fig. 1). This may be due to the compact space within the spinal canal in the mid-thoracic region. A compact spinal canal provides a limited operative space, resulting in a higher likelihood of inadvertent violation to the dural sac during surgery.

Currently, there are no convincing explanations for the associations between some of the clinical parameters and CSFL, such as being female or having hypertension and diabetes mellitus (Table 4). However, the identification of the three essential risk factors for CSFL might assist in the understanding of these unusual risk factors in a future study. As other studies have demonstrated gender disparity in prevalence of OPLL [21-23], we noted that female patients in our cohort were also more likely to have OPLL. We speculated that this might be the underlying reason for the higher association between females and higher incidence of CSFL (Table 4). Also as hypertension and diabetes mellitus are age-related conditions and older age was a significant risk factor for CSFL (Table 4), this association may explain the increased risk for CSFL in this population of patients. A recent large-scale survey confirmed that diabetes mellitus was a predisposing factor for OPLL [23], and this was compatible with our finding. Furthermore, patients with lower preoperative JOA scores tended

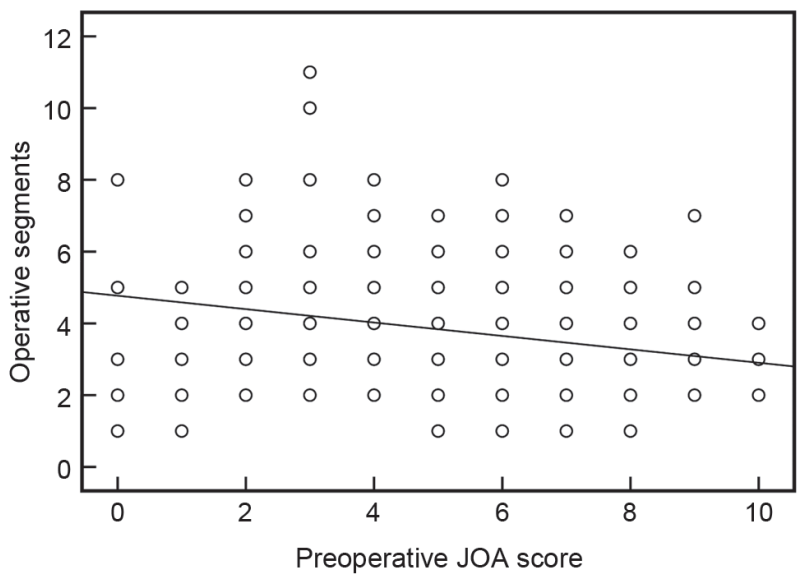

Fig. 2. Diagram displaying the relationship between preoperative JOA scores and operative segments in this cohort. The trend line indicates that operative segments negatively correlated with preoperative JOA scores ( $r=-0.272, p<0.05$, two-tailed Spearman's correlation test). JOA, Japanese Orthopaedic Association.

to have longer operative segments (Fig. 2), and this might result in the increased incidence of CSFL for the subgroup with preoperative JOA scores lower than 7.

In this study, only 5 cases of additional thoracic decompressions involved manipulations on formerly operated segments, and all of them developed CSFL. Thus, revision surgery was a significant risk factor for CSFL, which has been repeated proven $[1,3,6,8]$. As statistical analysis with such few subjects is not appropriate, we did not specifically discuss it in this present study. There were also some limitations to this study: Firstly, this study was performed in a single institution, though it harbored a large sample size; as such, a multi-institutional study may be required to validate our findings. Secondly, this study was a retrospective study and its findings need to be examined this characteristic in mind. Finally, though we followed previous studies to establish the determining criteria for CSFL $[6,12]$, these criteria were indirect rather than being direct evidence of CSFL. This limitation might affect the statistical results in this study.

\section{Conclusions}

Through recruiting a total of 362 cases, this study found that $32.3 \%$ of the thoracic decompressions performed were complicated with CSFL. Though many clinical factors were associated with CSFL, presence of OPLL, older age and longer operative segments were the most significant predisposing factors. In the light of the finding that 
the CD procedures had the highest incidence of CSFL, spinal surgeons should be mindful of this added risk performing $\mathrm{CD}$ procedure. This also applies to mid-thoracic decompression operations, as they also carried an additional risk for CSFL.

\section{Conflict of Interest}

No potential conflict of interest relevant to this article was reported.

\section{Acknowledgments}

We hereby thank and acknowledge the support of the Capital Foundation of Characteristic Clinical Practice and Research (Grant No. Z141107002514011) and the Foundation of Science and Research Projects for Capital Health Development (Grant No. 2014-2-4094) towards this study.

\section{References}

1. Hannallah D, Lee J, Khan M, Donaldson WF, Kang JD. Cerebrospinal fluid leaks following cervical spine surgery. J Bone Joint Surg Am 2008;90:1101-5.

2. Syre P, Bohman LE, Baltuch G, Le Roux P, Welch WC. Cerebrospinal fluid leaks and their management after anterior cervical discectomy and fusion: a report of 13 cases and a review of the literature. Spine (Phila Pa 1976) 2014;39:E936-43.

3. Khan MH, Rihn J, Steele G, et al. Postoperative management protocol for incidental dural tears during degenerative lumbar spine surgery: a review of 3,183 consecutive degenerative lumbar cases. Spine (Phila Pa 1976) 2006;31:2609-13.

4. Nandyala SV, Elboghdady IM, Marquez-Lara A, Noureldin MN, Sankaranarayanan S, Singh K. Cost analysis of incidental durotomy in spine surgery. Spine (Phila Pa 1976) 2014;39:E1042-51.

5. Yoshihara H, Yoneoka D. Incidental dural tear in spine surgery: analysis of a nationwide database. Eur Spine J 2014;23:389-94.

6. Baker GA, Cizik AM, Bransford RJ, et al. Risk factors for unintended durotomy during spine surgery: a multivariate analysis. Spine J 2012;12:121-6.

7. Weber C, Piek J, Gunawan D. Health care costs of incidental durotomies and postoperative cerebrospinal fluid leaks after elective spinal surgery. Eur Spine J
2015;24:2065-8.

8. Cammisa FP Jr, Girardi FP, Sangani PK, Parvataneni HK, Cadag S, Sandhu HS. Incidental durotomy in spine surgery. Spine (Phila Pa 1976) 2000;25:2663-7.

9. Yamazaki M, Okawa A, Fujiyoshi T, Furuya T, Koda M. Posterior decompression with instrumented fusion for thoracic myelopathy caused by ossification of the posterior longitudinal ligament. Eur Spine J 2010; 19:691-8.

10. Min JH, Jang JS, Lee SH. Significance of the doubleand single-layer signs in the ossification of the posterior longitudinal ligament of the thoracic spine. Neurosurgery 2007;61:118-21.

11. Matsumoto M, Chiba K, Toyama Y, et al. Surgical results and related factors for ossification of posterior longitudinal ligament of the thoracic spine: a multiinstitutional retrospective study. Spine (Phila $\mathrm{Pa}$ 1976) 2008;33:1034-41.

12. Sun $X$, Sun C, Liu X, et al. The frequency and treatment of dural tears and cerebrospinal fluid leakage in 266 patients with thoracic myelopathy caused by ossification of the ligamentum flavum. Spine (Phila Pa 1976) 2012;37:E702-7.

13. Hu P, Yu M, Liu X, Liu Z, Jiang L. A circumferential decompression-based surgical strategy for multilevel ossification of thoracic posterior longitudinal ligament. Spine J 2015;15:2484-92.

14. Tsuzuki N, Hirabayashi S, Abe R, Saiki K. Staged spinal cord decompression through posterior approach for thoracic myelopathy caused by ossification of posterior longitudinal ligament. Spine (Phila $\mathrm{Pa}$ 1976) 2001;26:1623-30.

15. Matsuyama Y, Yoshihara H, Tsuji T, et al. Surgical outcome of ossification of the posterior longitudinal ligament (OPLL) of the thoracic spine: implication of the type of ossification and surgical options. J Spinal Disord Tech 2005;18:492-7.

16. Aizawa T, Sato T, Sasaki H, et al. Results of surgical treatment for thoracic myelopathy: minimum 2-year follow-up study in 132 patients. J Neurosurg Spine 2007;7:13-20.

17. Yamazaki M, Mochizuki M, Ikeda Y, et al. Clinical results of surgery for thoracic myelopathy caused by ossification of the posterior longitudinal ligament: operative indication of posterior decompression with instrumented fusion. Spine (Phila Pa 1976) 2006;31: 1452-60. 
18. Kawahara N, Tomita K, Murakami H, et al. Circumspinal decompression with dekyphosis stabilization for thoracic myelopathy due to ossification of the posterior longitudinal ligament. Spine (Phila Pa 1976) 2008;33:39-46.

19. Liu FJ, Chai Y, Shen Y, Xu JX, Du W, Zhang P. Posterior decompression with transforaminal interbody fusion for thoracic myelopathy due to ossification of the posterior longitudinal ligament and the ligamentum flavum at the same level. J Clin Neurosci 2013; 20:570-5.

20. Sanghvi AV, Chhabra HS, Mascarenhas AA, Mittal VK, Sangondimath GM. Thoracic myelopathy due to ossification of ligamentum flavum: a retrospective analysis of predictors of surgical outcome and factors affecting preoperative neurological status. Eur Spine J
2011;20:205-15.

21. Mori K, Imai S, Kasahara T, Nishizawa K, Mimura T, Matsusue Y. Prevalence, distribution, and morphology of thoracic ossification of the posterior longitudinal ligament in Japanese: results of CT-based crosssectional study. Spine (Phila Pa 1976) 2014;39:394-9.

22. Sohn S, Chung CK, Yun TJ, Sohn CH. Epidemiological survey of ossification of the posterior longitudinal ligament in an adult Korean population: threedimensional computed tomographic observation of 3,240 cases. Calcif Tissue Int 2014;94:613-20.

23. Fujimori T, Le $\mathrm{H}, \mathrm{Hu} \mathrm{SS}$, et al. Ossification of the posterior longitudinal ligament of the cervical spine in 3161 patients: a CT-based study. Spine (Phila Pa 1976) 2015;40:E394-403. 